\title{
Desafios na redução da mortalidade materna no Município de São Paulo
}

\author{
Chalenges to reduce maternal mortality in São Paulo City
}

\section{Carlos Eduardo Pereira Vega}

Vega CEP. Desafios na redução da mortalidade materna no Município de São Paulo/ Chalenges to reduce maternal mortality in São Paulo City. Rev Med (São Paulo). 2018 mar.-abr.;97(2):235-43.

RESUMO: Desde 1993 as causas da mortalidade materna vem sendo estudadas pelo Comitê de Mortalidade Materna do Município de São Paulo. Até o ano de 2016 acumulamos 3505 mortes maternas, sendo 2389 óbitos devidos a causas diretas ou indiretas. A principal causa da mortalidade materna é a hipertensão arterial, com 538 (22,5\%) óbitos, seguida pelas complicações hemorrágicas de terceiro trimestre e puerpério com $330(13,8 \%)$ ocorrências. As outras três causas responsáveis pelo óbito materno são as doenças cardiovasculares, complicações de aborto (incluindo os abortos inseguros) e a infecção puerperal. São feitas considerações sobre as cinco principais causas de morte materna e são disponibilizadas sugestões elaboradas pelo Comitê de Mortalidade Materna do Município de São Paulo para a redução desses eventos. As demais causas em menor número, mas não menos importantes, não serão objeto desse estudo.

Descritores: Mortalidade materna; Hipertensão induzida pela gravidez; Hemorragia pós-parto; Complicações na gravidez; Aborto; Infecção puerperal.

\section{INTRODUÇÃO}

$\mathrm{D}$ esde a sua criação em 21 de dezembro de 1992', o Comitê de Mortalidade Materna do Município de São Paulo (CMMMSP) vem desempenhando um trabalho contínuo na busca de casos positivos ou presumíveis de envolvimento com a morte materna, bem como estabelecendo propostas para a sua redução.

A expectativa pela redução dos eventos de morte materna e suas graves consequências foi sendo minimizada
ABSTRACT: Since 1993 the causes of maternal mortality has been studied by The Maternal Mortality Committee of the municipality of São Paulo. By the year 2016 the city accumulates 3505 maternal deaths, and 2389 are due to direct or indirect causes. The main cause of maternal mortality is high blood pressure, with $538(22.5 \%)$ deaths, followed by bleeding complications of third quarter and puerperium with $330(13.8 \%)$ instances. The other three main causes for maternal death are cardiovascular diseases, miscarriage (including unsafe abortions) and puerperal infection. The Maternal Mortality Committee of the municipality of São Paulo makes considerations about the five leading causes of maternal death and are offer suggestions to reduce these events. The other causes, with fewer, but no less important, are not object of this study.

KEYWORDS: Maternal mortality; Hypertension, pregnancyinduced; Postpartum hemorrhage; Pregnancy complications; Abortion; Puerperal infection.

com a obtenção de resultados positivos. Entretanto, ainda estamos muito distantes do nosso ideal e muito trabalho se apresenta para o futuro. No decorrer desse trabalho de pesquisa, foi-se firmando um diagnóstico das principais causas determinantes do óbito materno em nossa cidade e um processo de elaboração de propostas de atuação foi tomando forma. O CMMMSP consegue, com grande confiabilidade, estabelecer um diagnóstico real da nossa situação frente a esse grave problema. Apesar dos problemas determinantes da morte materna já serem

Comitê de Mortalidade Materna do Município de São Paulo. Secretaria da Saúde do Município de São Paulo. Email: carlosvega@ prefeitura.sp.gov.br

Endereço para correspondência: Rua General Jardim, 36 - 5º andar. São Paulo, SP, Brasil. CEP: 01223-010. 
Vega CEP. Desafios na redução da mortalidade materna no Município de São Paulo.

conhecidos há muito tempo, foi necessária a formação de uma casuística consistente e específica para a cidade de São Paulo para termos ciência da real dimensão do problema envolvendo a morte materna. Ressaltamos que esse estudo deve ser específico para cada município, pois uma patologia comum a dois municípios pode ser determinada por fatores distintos.

Muitas pessoas se engajaram nessa luta mesmo antes da existência oficial do Comitê. Muitas se somaram a esse grupo, que hoje tem pessoal treinado e capacitado para desempenhar o papel de busca e análise dos casos de morte materna com segurança.

Entretanto, reduzir a morte materna não é um problema fácil de resolver. O óbito materno é determinado por uma série de fatores que perpassam pela dificuldade de acesso à educação, pelos serviços de saúde e a bens de consumo, envolve questões étnico-raciais e abrange falhas no atendimento propriamente dito.

Essa pesquisa abrangendo quase 25 anos de estudo da morte materna, suas determinantes e suas consequências, não tem como objetivo expor dados detalhados e sim, fornecer uma visão geral do que foi apurado pelo trabalho ininterrupto exercido pelo Comitê de Mortalidade Materna e seus membros. Dados mais apurados poderão ser obtidos em consulta as nossas outras publicações ${ }^{2}$.

Dada a dimensão da cidade de São Paulo, o Comitê Municipal é composto por um Comitê Central e 25 Comitês Locais distribuídos em todas as regiões da nossa cidade e, se não fosse o trabalho árduo exercido por esses Comitês, muitas vezes utilizando recursos próprios para executar a contento suas responsabilidades, o resultado obtido nunca seria alcançado.

Longe de se ater às regras que balizam uma publicação científica, essa revisão efetua uma comparação histórica do comportamento das principais patologias determinantes da mortalidade materna e suas nuances em nossa cidade, servindo apenas para termos uma visão geral do problema. Porém, esse trabalho não contribuiria em nada se não apresentasse o caminho que trilhamos para a obtenção dos resultados alcançados e estabelecesse propostas para a redução do indicador. É o que veremos a seguir.

\section{METODOLOGIA}

Esse estudo utilizou os dados apurados nas investigações de casos de morte materna levando-se em consideração o que foi identificado nas visitas domiciliar e hospitalar, informações do Serviço de Verificação de Óbitos da Capital (SVOC), Instituto Médico Legal (IML), site de Notificação de Óbitos Maternos, cruzamento de Declarações e informações leigas.

Alguns óbitos maternos de mulheres residentes aconteceram fora do nosso município e gostaríamos de agradecer aos Comitês de Mortalidade Materna dos
Municípios da Grande São Paulo que providenciaram a troca de informações necessárias para a conclusão desses casos.

Desde a publicação da Portaria 1.119 de 05 de junho de 2008, que obriga a todos os Comitês do Brasil a investigar todos os óbitos ocorridos em mulheres em idade fértil, nossa preocupação tem sido em atingir uma meta numérica onde não se perca qualidade em detrimento da quantidade.

O município de São Paulo não pode ser comparado a nenhuma outra cidade do nosso país. Com população numericamente semelhante ao do Estado do Paraná e maior do que a soma de sete Estados brasileiros mais o Distrito Federal, não pode ter o mesmo tratamento no que tange às normas.

Dessa forma, desenvolvemos uma metodologia própria de trabalho que se mostrou eficaz e muito abrangente na identificação e coleta de casos de morte materna.

Utilizamos como base o Método de Máscaras, que consiste na identificação de uma série de patologias que podem complicar ou serem complicadas pelo estado gestacional. Esse processo é utilizado desde a implantação do Comitê e tem por base orientações do próprio Ministério da Saúde ${ }^{3}$. Entretanto, com o passar dos anos, verificamos que essa metodologia, embora muito eficaz, ainda deixava a desejar. Acrescentamos mais alguns eventos à lista original de patologias, mas sem obter o resultado desejado.

No ano de 2005 agregamos mais uma ferramenta para identificar casos positivos para morte materna. Criamos um link na nossa página da Internet ${ }^{4}$ para que qualquer munícipe ou profissional de saúde tenha a possibilidade de notificar a ocorrência de um caso sabidamente positivo. A ficha gerada pelo sistema desencadeia um e-mail dirigido a uma caixa postal específica, contendo o nome, data e endereço da falecida, o que agiliza o processo de investigação. Esse link continua em atividade até o momento e tem contribuído para a identificação precoce dos óbitos maternos.

Ainda em 2005, não satisfeitos com os resultados obtidos, iniciamos um processo de cruzamento das Declarações de Óbito de mulheres em idade fértil com as Declarações de Nascido, procurando congruências. Esse cruzamento se mostrou bastante eficaz, principalmente na identificação de óbitos ocorridos após o $42^{\circ}$ dia de puerpério, onde a interferência do ciclo gravídico-puerperal e suas implicações se tornam mais tênues.

Cabe aqui a ressalva de que a utilização do Método de Mascaras de forma isolada identifica mais de $90 \%$ dos casos diretos ou indiretos de morte materna até 42 dias do parto ou aborto. As outras metodologias agregadas apenas aprimoram esses valores e afloram mais os casos não obstétricos bem como algumas mortes maternas tardias.

Para efeito de comparação, utilizamos o indicador Razão de Mortalidade Materna (RMM) que consiste 
na divisão do número de óbitos maternos diretos (CID$10=000.0-095)$ ou indiretos (CID-10=O98.0-O99.8) pelo número de nascidos vivos ocorridos no período estudado.

A classificação correta de cada caso permite deslumbrar o real perfil da mortalidade materna. Para o Comitê Municipal isso somente é possível após uma exaustiva investigação abrangendo a visita hospitalar, domiciliar e laudos do SVOC ou do IML. Com base nas informações colhidas, o caso é discutido com profissionais altamente treinados em classificação e codificação, tornando o resultado obtido muito mais confiável.

Dada à inexistência de recursos específicos destinados ao trabalho dos Comitês, que se obrigam a utilizar a estrutura já existente do sistema de saúde para promover seu trabalho investigativo, não vemos como produtivo a investigação de todos os óbitos de mulheres em idade fértil. Acreditamos que nossa metodologia possa ser empregada em cidades de grande porte sem incorrer na perda significativa de informações.

\section{RESULTADOS E CONSIDERAÇÕES}

\section{Perfil geral da mortalidade materna}

Durante esses anos de existência do Comitê Municipal, acumulamos a triste marca de 3505 óbitos maternos em mulheres residentes em nossa cidade. Excluímos 26 óbitos maternos ocasionados pela pandemia por Influenza A (H1N1) ocorrida em 2009 e 2010 por entendermos que uma pandemia foge do padrão habitual e pode determinar erros de interpretação de resultados em uma série histórica.

Entretanto, para não deixarmos de lado essa calamidade mundial, divulgamos uma pesquisa específica sobre as ocorrências da Influenza A (H1N1) e seu impacto na mortalidade materna em nosso município 5 .

O conceito clássico da Razão de Mortalidade Materna (RMM) se traduz pela soma dos casos Diretos e Indiretos dividido pelo número de Nascidos Vivos durante o período estudado, sendo o resultado multiplicado por 100.000 para dar visibilidade ao indicador. Entretanto, como podemos verificar no Gráfico 1, dada a grande variação anual da RMM, a visualização da tendência do indicador deixa muito a desejar. Dessa forma, para termos uma noção evolutiva do resultado dos esforços empregados, disponibilizamos no mesmo Gráfico o comportamento trienal do indicador, padrão que adotaremos neste estudo.

Os 3505 casos positivos para morte materna ocorridos em mulheres residentes no Município de São Paulo foram agrupados na Tabela 1, segundo a classificação preconizada pela CID-10.

Gráfico 1. Distribuição da Razão de Mortalidade Materna Anual e Trienal - MSP - 1993-2016

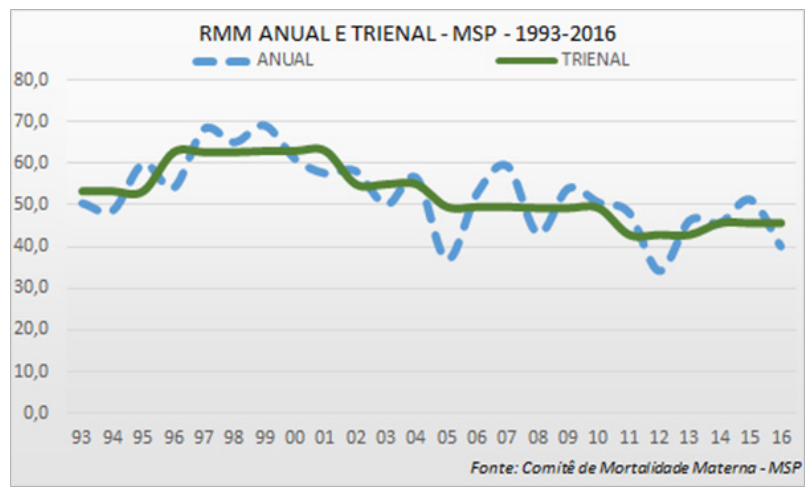

Tabela 1. Distribuição dos 3505 casos de morte materna de mulheres residentes no município de São Paulo - 1993-2016

\begin{tabular}{llllllllll}
\hline Causas & $\mathbf{9 3 - 9 5}$ & $\mathbf{9 6 - 9 8}$ & $\mathbf{9 9 - 0 1}$ & $\mathbf{0 2 - 0 4}$ & $\mathbf{0 5 - 0 7}$ & $\mathbf{0 8 - 1 0}$ & $\mathbf{1 1 - 1 3}$ & $\mathbf{1 4 - 1 6}$ & Total \\
\hline Diretas & 192 & 200 & 195 & 150 & 133 & 123 & 120 & 119 & 1232 \\
Indiretas & 112 & 208 & 193 & 154 & 127 & 134 & 105 & 124 & 1157 \\
Tardias & 0 & 16 & 36 & 45 & 41 & 41 & 34 & 20 & 233 \\
Não relacionadas & 7 & 18 & 24 & 37 & 25 & 26 & 26 & 28 & 191 \\
Não relacionadas tardias & 1 & 43 & 66 & 76 & 106 & 124 & 110 & 166 & 692 \\
Total & $\mathbf{3 1 2}$ & $\mathbf{4 8 5}$ & $\mathbf{5 1 4}$ & $\mathbf{4 6 2}$ & $\mathbf{4 3 2}$ & $\mathbf{4 4 8}$ & $\mathbf{3 9 5}$ & $\mathbf{4 5 7}$ & $\mathbf{3 5 0 5}$ \\
\hline
\end{tabular}

Fonte: Comitê de Mortalidade Materna - MSP

Como nosso foco de estudo abrange os casos diretos e indiretos, usaremos como base 2389 casos e, para não nos alongarmos demais, vamos tecer considerações apenas sobre as cinco principais patologias determinantes do óbito materno.

Cada patologia responsável pelo óbito materno possui características singulares que nos permitem elaborar propostas de redução mais consistentes.

Cabe aqui a ressalva de que nosso estudo é baseado em informações coletadas em prontuários médicos, antecedentes familiares e laudo de necropsia, o que limita muito uma classificação mais apurada de alguns casos, dada a falta de subsídios para tal.

\section{Hipertensão arterial}

A hipertensão arterial foi responsável por 538 (22,5\%) dos óbitos maternos de 1993 a 2016², sendo 334 
Vega CEP. Desafios na redução da mortalidade materna no Município de São Paulo.

(62,1\%) dos óbitos causados pela pré-eclâmpsia/eclâmpsia (PE/E) e os 204 (37,9\%) restantes pela hipertensão arterial sistêmica (HAS).

O Gráfico 2 apresenta a série histórica da RMM por hipertensão arterial com os dois componentes estudados.

Gráfico 2. Série histórica da Razão de Mortalidade Materna por Hipertensão Arterial e seus componentes - MSP - 1993-2016

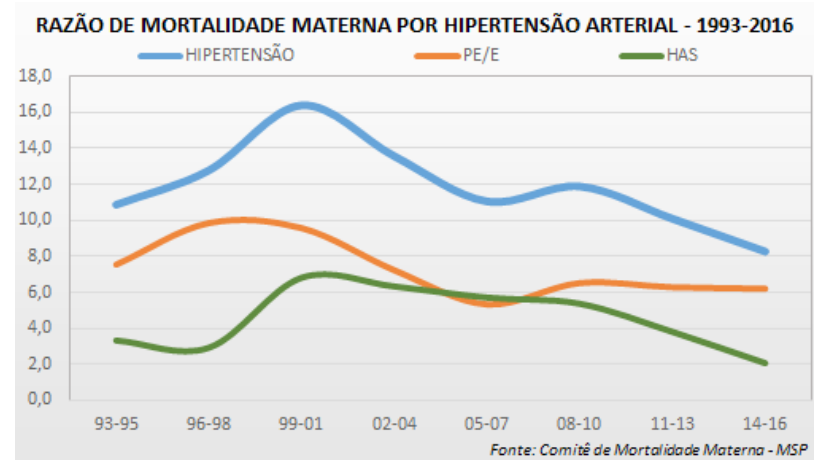

Apesar de ambas determinarem o óbito materno, a abordagem para redução dos eventos difere em alguns pontos.

Há anos se debate a prevenção da pré-eclâmpsia/ eclâmpsia. A identificação da gestante portadora de fatores de risco (obesidade, gemelaridade, antecedentes obstétricos, pressão arterial, diabetes, alterações ultrassonográficas, raça, etc. $)^{6}$ para o desenvolvimento da pré-eclâmpsia é imperiosa.

Entretanto, ações preventivas como o uso do ácido acetilsalicílico ${ }^{7}$ e a reposição de cálcio ${ }^{8}$ somente surtem efeito em gestantes portadoras de fatores de risco, sendo infrutíferas na população em geral.

Dessa forma, um acompanhamento pré-natal de qualidade, onde a identificação dos fatores de risco e a adoção das medidas preventivas apropriadas a cada caso é benéfico para a prevenção desses eventos.

Por outro lado, o acompanhamento da mulher portadora de hipertensão arterial sistêmica deve se iniciar antes do início da gravidez.

Uma estabilização pressórica prévia à gestação e a adequação da medicação em uso, evitando-se o uso de inibidores da enzima conversora de angiotensina (captopril, enalapril, etc. $)^{9}$, se traduz em benefício certo para a evolução da gestação à contento.

Como se sabe, a mortalidade materna tem um vínculo inversamente proporcional ao grau de desenvolvimento sócio-econômico de uma população, ou seja, quanto menor o desenvolvimento de uma sociedade, maior a Razão de Mortalidade Materna. No estudo da hipertensão arterial isso se torna bem evidente.

O Mapa 1 mostra a distribuição da RMM determinada pela hipertensão arterial nas diversas regiões do MSP no último triênio (esquerda) comparada com o mapa do Índice Paulista de Vulnerabilidade Social (IPVS) de 2010.

Mapa 1. Distribuição geográfica da Razão de Mortalidade Materna por Hipertensão Arterial (2014-2016) e do Índice Paulista de Vulnerabilidade Social (2010)

RMM HIPERTENSÃO 2014-2016

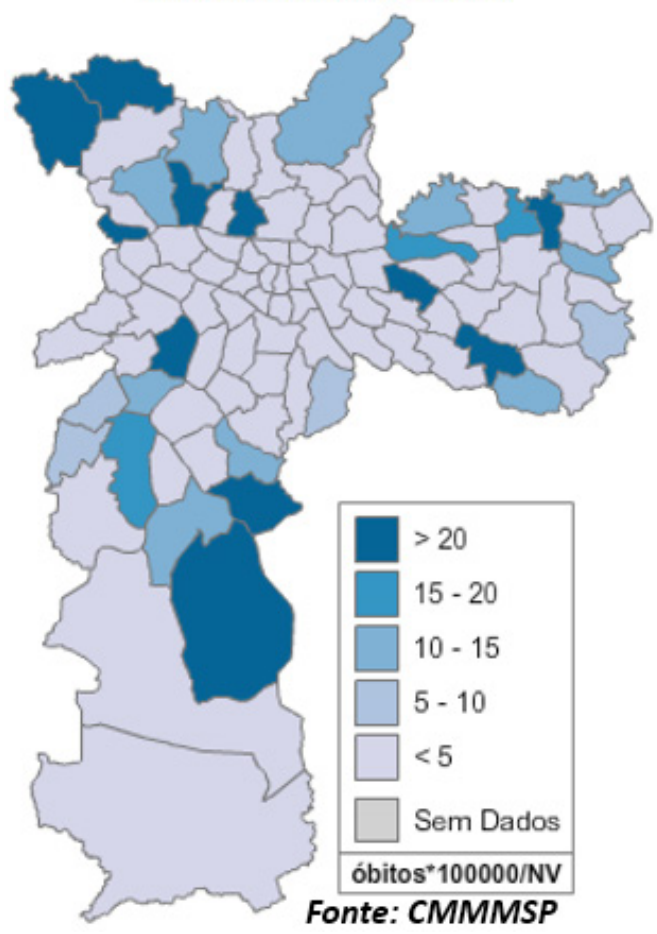

Índice Paulista de Vulnerabilidade Social (IPVS) Municipio de São Paulo - 2010

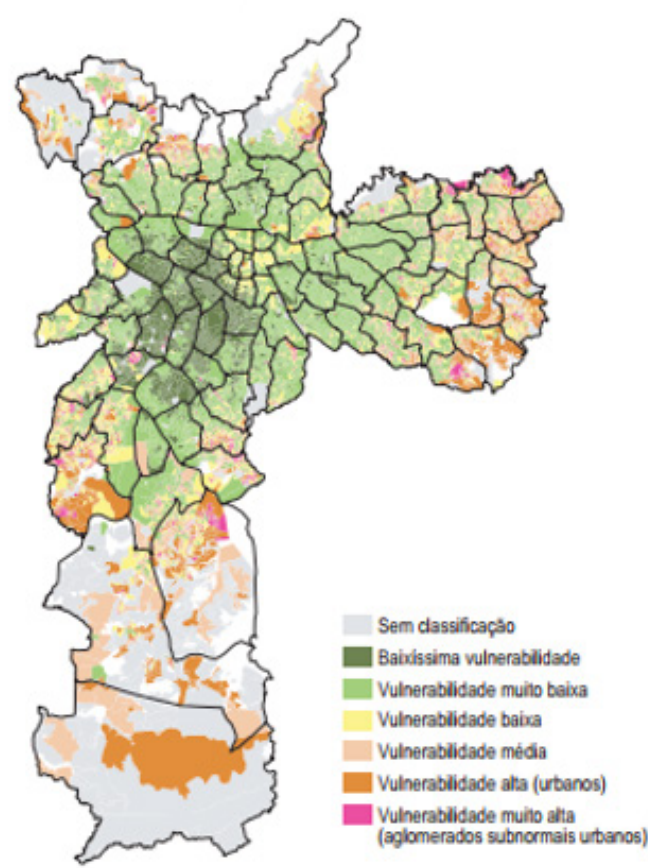

Fonte: Fundaçåo Seade. Índice Paulista de Vulnerabilidade Social - IPVS. 
Como podemos verificar, é na periferia da cidade que encontramos as maiores RMM.

A baixa escolaridade, o baixo poder aquisitivo e a dificuldade de acesso à saúde de qualidade são fatores que podem influenciar no aumento da morbidade determinada pela hipertensão arterial, levando a consequências trágicas.

$\mathrm{O}$ atendimento a essa população vulnerável exige um pré-natal de início precoce, grupos de gestantes, orientação adequada para uso da medicação prescrita e a necessidade da sua utilização para um melhor controle pressórico.

É fundamental a disponibilização dos medicamentos anti-hipertensivos pois sabemos que a aquisição dos medicamentos não é prioridade quando se trata de população carente.

A hipertensão em si não determina o óbito materno, mas sim, as complicações advindas do descontrole pressórico.

Durante praticamente toda nossa série histórica verificamos que o acidente vascular cerebral despontava como primeira causa do óbito materno determinada pela hipertensão arterial. Entretanto, nos últimos três anos, os quadros hemorrágicos (síndrome HELLP, rotura hepática, descolamento prematuro de placenta) predominaram. Em terceiro lugar encontramos o edema agudo de pulmão.

Para termos noção da gravidade o quadro, $50 \%$ dos óbitos por acidente vascular cerebral ocorreram até o primeiro dia após a instalação do quadro. Dessa forma, é fundamental a prevenção da crise convulsiva ou de suas recorrências. Na iminência de eclâmpsia devemos, obrigatoriamente, lançar mão do sulfato de magnésio. Medicamento de baixo custo e de manuseio relativamente fácil, é a droga de escolha para prevenção da crise convulsiva e suas consequências.

No município de São Paulo a utilização dessa medicação vem aumentando consideravelmente e, talvez, explique a redução do número de óbitos por acidente vascular cerebral verificada nos últimos seis anos.

$\mathrm{Na}$ análise das complicações hemorrágicas identificamos um predomínio da síndrome HELLP e da rotura hepática.

A atenção obstétrica deve ser redobrada nessas situações, pois, após a instalação da coagulopatia, a morbidade e a mortalidade materna aumentam consideravelmente ${ }^{10}$. A reposição de hemoderivados (concentrado de hemáceas, plasma fresco, plaquetas e fatores de coagulação) requer um Banco de Sangue ágil e bem abastecido desses produtos.

A rotura hepática se faz de maneira silenciosa e muitas vezes surpreende a equipe obstétrica.

Esses casos mais complexos devem ser abordados sempre com uma equipe multiprofissional e multidepartamental habilitada no manuseio dessas emergências.

Fica claro que não devemos esperar a instalação do processo de coagulopatia para se tomar uma providência. $\mathrm{O}$ controle laboratorial é importante e a agilidade na tomada de conduta é imperiosa. Entretanto, condutas intempestivas podem levar a danos em outros órgãos, aumentando a morbidade e a mortalidade.

O manuseio da reposição volêmica deve ser feito por equipe especializada, levando-se em conta a pressão venosa central, pois a administração de colóides de forma indiscriminada pode desencadear o edema agudo de pulmão.

Apesar de a grande maioria das complicações cessar após a retirada da placenta, os quadros clínicos mais drásticos perpetuam por vários dias, exigindo uma supervisão constante.

Outro dilema enfrentado pelo obstetra é a intervenção versus bem-estar fetal. A linha que divide os dois sistemas é muito tênue e todo cuidado é pouco para a obtenção de resultados satisfatórios para ambas as partes.

O caminho para redução da mortalidade materna por hipertensão arterial é árduo e os resultados são parcos. Necessita um empenho de todas as esferas de atendimento, que devem unir esforços em prol de um objetivo comum.

\section{Hemorragia de final de gestação e puerpério}

A hemorragia é a segunda causa determinante do óbito materno em nosso município. Ao contrário da mortalidade materna por hipertensão arterial que avalia a atenção primária englobando o acompanhamento pré-natal, a mortalidade por hemorragia analisa o atendimento e a estrutura hospitalar.

Dentre os quadros hemorrágicos englobamos a atonia uterina, o acretismo placentário, o descolamento prematuro de placenta, a placenta prévia, a rotura uterina, além de outros menos frequentes.

O Gráfico 3 apresenta a evolução da Razão de Mortalidade Materna por hemorragia de $2^{\circ} / 3^{\circ}$ trimestres e puerpério durante o período estudado.

Gráfico 3. Série histórica da Razão de Mortalidade Materna por Hemorragias de $2^{\circ} / 3^{\circ}$ trimestres de gestação e puerpério - MSP $-1993-2016$

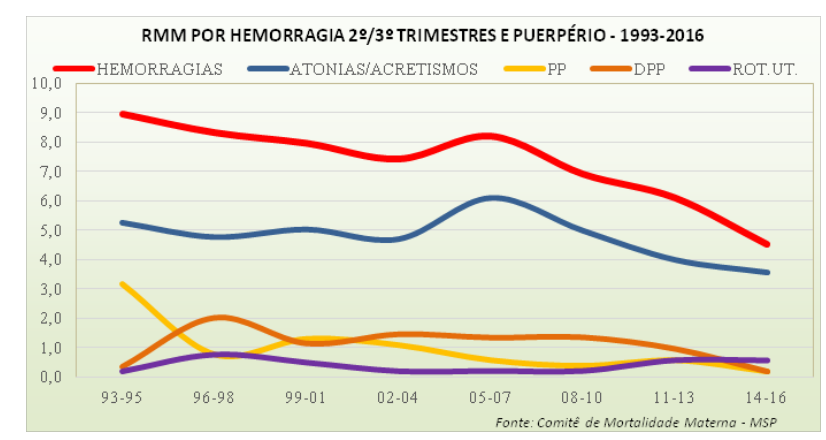


Vega CEP. Desafios na redução da mortalidade materna no Município de São Paulo.

A atonia uterina e o acretismo placentário (associados ou não) respondem pela principal causa de óbito por hemorragia e a maior parte dos óbitos decorrentes de atonia acontece em até seis horas após o parto.

Não podemos nos esquecer de que o tempo é nosso inimigo quando estamos diante de um quadro hemorrágico. As grandes perdas sanguíneas induzem às coagulopatias de consumo e o tratamento fica cada vez mais difícil. Na nossa casuística, 75,4\% dos óbitos por hemorragia aconteceram até o primeiro dia de puerpério.

Coligado diretamente aos quadros de atonia uterina, o acretismo placentário está diretamente relacionado à paridade, ao número de cesáreas e ao tabagismo ${ }^{11-13}$, devendo o obstetra estar atento a esses fatores predisponentes.

Devemos recomendar abstinência ao cigarro durante toda a gestação e evitar a realização da primeira cesárea, principalmente nas pacientes jovens, com um futuro obstétrico pela frente. A gravidez em tenra idade predispõe à ocorrência de comorbidades, expondo um organismo ainda em formação às mais diversas complicações obstétricas.

No último triênio verificamos que o setor privado foi responsável por $60,9 \%$ das cesáreas realizadas no nosso município e $82,7 \%$ quando analisado individualmente.

Segundo dados do Sistema de Informação sobre Nascidos Vivos (SINASC), a taxa de realização de cesáreas em adolescentes (abaixo de 20 anos) nos últimos três anos de abrangência desse estudo (2014-2016) foi de $23,3 \%$ no setor público contra 73,3\% no setor privado.

Apesar das múltiplas variáveis presentes nos estudos envolvendo a gravidez na adolescência existe um consenso: é fundamental a orientação específica para esse grupo voltada para a saúde sexual e reprodutiva. Mesmo que, em um primeiro momento, não se consiga a redução do número de gestações indesejadas nessa faixa etária, é fundamental que todos os adolescentes sejam acolhidos e orientados da melhor forma possível. Se ocorrer a gestação, o atendimento diferenciado desse grupo pode reduzir os medos e as ansiedades decorrentes da primeira gestação em um organismo ainda em formação, tornando a adolescente mais colaborativa e predisposta ao parto normal. A presença de um(a) acompanhante bem orientado(a) durante o trabalho de parto traz conforto e segurança para a parturiente, tornando o processo de parto mais fácil.

As modificações gravídicas (locais e sistêmicas) tornam a obstetrícia uma especialidade onde os sangramentos estão sempre presentes, em menor ou maior proporção. Dessa forma, toda maternidade deve ter na sua retaguarda um banco de sangue ágil e resolutivo para atender a uma demanda de emergência. Não raros são os casos onde o óbito foi decorrente da demora na reposição de hemoderivados, não disponíveis em tempo hábil.

A conduta obstétrica deve ser mais agressiva frente a um quadro hemorrágico. Todavia, não podemos deixar que a pressa interfira nos protocolos assistenciais ou no rigor que a técnica cirúrgica exige. Encontramos várias iatrogenias causando aumento da morbidade e, em algumas vezes, determinando o óbito. Tais iatrogenias foram decorrentes tanto da má condução do trabalho de parto como da execução dos procedimentos cirúrgicos correlatos ao atendimento (hematomas sub e supra-aponeuróticos, sangramentos de histerorrafia, hematomas de episiotomia, lacerações arteriais e vesicais, etc.) colaborando diretamente para o óbito materno.

Entretanto, ainda estamos muito aquém da medicina praticada nos países desenvolvidos. A identificação antenatal de um quadro de acretismo placentário é prioridade para se traçar um plano terapêutico visando à prevenção de complicações ${ }^{14}$. A utilização da radiologia intervencionista deve ser prática habitual nesses casos, fato ainda restrito a poucos serviços e muito distante do nosso dia-a-dia.

Em situações onde o diagnóstico de acretismo placentário é feito a contento, pode-se planejar o parto com mais segurança, utilizando-se de equipe multidisciplinar com conhecimento profundo da anatomia pélvica, suporte da radiologia intervencionista e da urologia.

\section{Doenças Cardiovasculares}

As patologias cardiovasculares respondem pela terceira causa de morte materna em nosso município.

Para efeito de classificação, subdividimos o Grupo das Cardiopatias em cinco subgrupos, a saber: as miocardiopatias de origem funcional (determinadas pela falência funcional do miocárdio - Insuficiência Cardíaca Congestiva), o infarto agudo do miocárdio, as valvulopatias (congênitas ou adquiridas), os aneurismas de aorta e as cardiopatias de origem infecciosa (doença de Chagas, cardites e pericardites).

Sob a ótica cardiovascular, a gestação saudável cursa com a harmônica interação de três fatores: queda na resistência vascular periférica (RVP), hipervolemia consequente à grande expansão do volume plasmático e hipercoagulabilidade ${ }^{15}$. As transformações no organismo materno objetivam sustentar o desenvolvimento fetal e têm início logo após a concepção.

Para fazer face à hipervolemia, o coração é instado a aumentar seu rendimento e o faz às custas da elevação da frequência cardíaca, que se inicia na $4^{\mathrm{a}}$ semana, tornando-se máxima entre a $28^{\mathrm{a}}$ e a $32^{\mathrm{a}}$ semanas, e do volume sistólico que atinge o seu maior valor na $32^{\mathrm{a}}$ semana, quando apresenta patamar $50 \%$ superior aos níveis iniciais, mantendo-se nestas cifras até o termo.

O trabalho de parto impõe maior esforço ao coração, aumentando em até $25 \%$ o seu rendimento. Em virtude das contrações uterinas que antecedem o parto e da redução volumétrica do útero no pós-parto imediato, a vasculatura uterina transfere à circulação sistêmica grande parte do volume sanguíneo nela contido, gerando abrupto incremento volêmico. Muitas vezes, a dor e a ansiedade, ao elevarem o tônus adrenérgico, acentuam a sobrecarga cardíaca ${ }^{16,17}$. 
Vega CEP. Desafios na redução da mortalidade materna no Município de São Paulo.

A literatura aponta que quase metade dos aneurismas rotos de aorta ocorridos em mulheres abaixo dos 40 anos acontecem durante a gravidez ${ }^{18}$. As mudanças fisiológicas impostas pela gravidez aumentam o risco de dissecção, principalmente em mulheres portadoras de distúrbios do tecido conectivo, síndromes genéticas (p.ex. Síndrome de Marfan) e alterações congênitas do coração. Uma orientação pré-concepcional adequada e um seguimento pré-natal rigoroso são medidas importantes para evitar o óbito materno.

Ao analisarmos o momento do óbito em relação ao parto, verificamos que $74(37,0 \%)$ mulheres faleceram antes de dar à luz, sendo $15(20,3 \%)$ no primeiro trimestre, $29(39,2 \%)$ no segundo trimestre e $30(40,5 \%)$ no terceiro trimestre. As $126(63,0 \%)$ pacientes restantes faleceram no puerpério, sendo a maioria (83-66,0\%) antes de completar o $8^{\circ}$ dia de pós-parto.

Dessa forma, fica claro que a orientação pré-concepcional, o acompanhamento rígido do pré-natal e uma atenção redobrada no puerpério imediato, evitando a alta precoce, é fundamental para prevenção do óbito materno nas pacientes portadoras de cardiopatias congênitas ou adquiridas.

\section{Complicações de aborto}

As complicações de aborto englobam uma série de patologias que acometem a primeira metade da gravidez. Dentre as mais comuns, podemos citar o aborto inseguro, o aborto retido, os quadros hemorrágicos (gravidez ectópica e hemorragias decorrentes do processo de abortamento), as doenças trofoblásticas (mola hidatiforme e coricarcinoma) e o aborto infectado.

Há pouco tempo atrás, as complicações de aborto ocupavam o terceiro lugar nas causas determinantes da mortalidade materna. Entretanto cedeu lugar às complicações cardiovasculares no último triênio (2014-2016).

Durante o período de estudo, identificamos 222 casos de óbito materno por complicações de aborto. Em termos numéricos, os casos de aborto inseguro (87 casos $39,0 \%$ ) se traduzem na principal causa. Cabe aqui a ressalva de que os casos de aborto infectado ( 40 casos - 18,0\%) e os casos de suspeita sem confirmação de manipulação prévia (20 - 9,0\%) não foram associados aos comprovadamente considerados inseguros, pois não foi possível identificar se houve ou não uma tentativa de interrupção voluntária da gravidez. Se esses casos fossem agregados aos primeiros, teríamos 147 (66,0\%) eventos onde a infecção e/ou manipulação prévia foi responsável pelo óbito materno.

A prática do aborto inseguro reflete uma carência no campo da saúde sexual e reprodutiva. Entretanto, apesar de ainda não erradicarmos esse problema, presenciamos uma queda importante dessa causa nos últimos anos (Gráfico 4).
Gráfico 4. Razão de Mortalidade Materna por abortamento segundo causa - MSP - 1993-2016

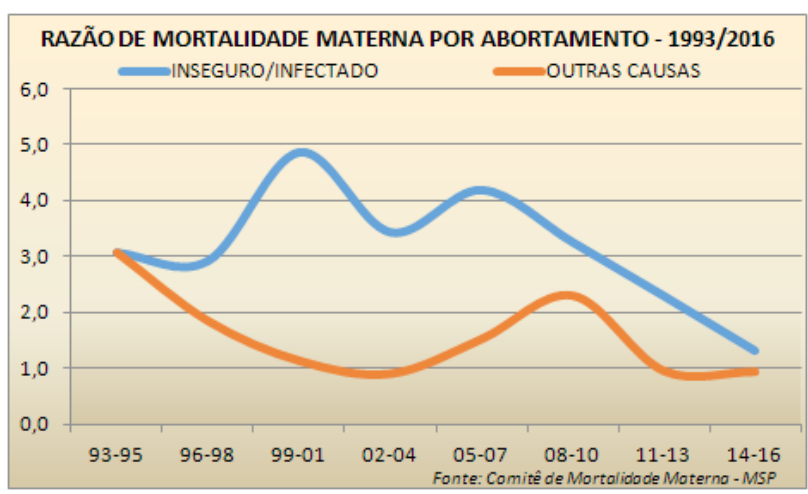

$\mathrm{O}$ advento das redes sociais, a maior liberdade de opinião e de expressão e as mudanças comportamentais no relacionamento sexual decorrente da utilização de métodos coadjuvantes de barreira para prevenção da AIDS/SIDA determinaram, principalmente na população mais jovem, mudanças importantes.

Entretanto, a pressão imposta pela sociedade obriga a adolescente a repensar o momento de engravidar e determina vários tipos de comportamento frente ao momento certo para assumir essa responsabilidade ${ }^{19}$.

Quanto às demais causas, a gravidez ectópica, a doença trofoblástica (mola e coriocarcinoma), o aborto retido e os abortos hemorrágicos respondem pela quase a totalidade dos casos restantes.

Independente da causa, a orientação adequada sobre a disponibilidade e utilização dos métodos anticoncepcionais, um pré-natal de início precoce e a realização de exames ultrassonográficos ao redor da $6^{\mathrm{a}}-8^{\mathrm{a}}$ semanas de gestação minimizariam muito essas ocorrências.

\section{Infecção puerperal}

A quinta causa de óbito materno em nosso município é a infecção puerperal. Responsável por 153 óbitos maternos, traduz o descuido com os Protocolos Assistenciais e com a tocurgia.

O SUS foi responsável pelo atendimento de 121 $(79,1 \%)$ casos contra $20(13,1 \%)$ ocorridos no setor privado. Nos $12(7,8 \%)$ casos restantes não foi possível determinar o local do óbito.

Entretanto, quando estudamos a infecção puerperal, nosso foco de interesse é o hospital onde ocorreu o parto e não o óbito, pois o atendimento ao parto é crucial na determinação da infecção puerperal. Desde 2001, o SINASC começou a tabular de forma mais consistente o tipo de parto segundo o hospital de ocorrência. Dessa forma, para melhor avaliação, restringiremos o estudo da mortalidade materna por infecção puerperal para esses últimos 16 anos. 
Vega CEP. Desafios na redução da mortalidade materna no Município de São Paulo.

Encontramos 82 óbitos maternos decorrentes da infecção puerperal de 2001 a 2016. Desses, 42 (51,2\%) casos foram submetidos à cesárea e $40(48,8 \%)$ evoluíram para parto vaginal.

O SUS realizou o parto em $67(81,7 \%)$ casos e o setor privado em $12(14,6 \%)$. Identificamos três $(3,7 \%)$ partos em domicílio.

Cabe aqui uma consideração: nesses 16 anos de estudo, o SINASC identificou a ocorrência de 9115 partos em domicílio, determinando uma RMM específica para infecção puerperal de 32,9/100.000 NV, muito além da RMM observada no atendimento hospitalar $(2,6 / 100.000 \mathrm{NV})$.

Quando calculamos a RMM específica para cada procedimento, ou seja, número de óbitos por infecção puerperal determinados pela cesárea divididos sobre o número de cesáreas realizadas no período (idem para parto vaginal), verificamos que a cesárea determinou uma RMM de 2,4 e o parto vaginal uma RMM de 2,8 .

Podemos inferir que tanto o parto vaginal quanto o operatório determinam valores semelhantes de infecção puerperal. Dessa forma, fica claro que, em ambas as situações, não houve respeito aos Protocolos Obstétricos nem o cuidado com a realização do procedimento em si.

O parto vaginal traz muitos benefícios para o binômio mãe-concepto. Entretanto, fica difícil estimular a realização do parto vaginal em detrimento do operatório nas condições atuais, onde a mortalidade por infecção puerperal nos dois procedimentos é praticamente a mesma.

É imperioso o exercício da obstetrícia na sua forma plena, com respeito aos protocolos e as técnicas empregadas.

\section{CONSIDERAÇÕES FINAIS}

Avaliando as cinco principais causas determinantes do óbito materno fica claro que muito pode ser feito para promover a redução dessas ocorrências.

Chegamos à conclusão de que a solução para a

\section{REFERÊNCIAS}

1. Câmara Municipal do Município de São Paulo. Lei Municipal 11.313 1992. Disponível em: http://www.prefeitura. sp.gov.br/cidade/secretarias/saude/saude_da_mulher/index. php? $\mathrm{p}=5778$.

2. Vega CEP. Relatórios de mortalidade materna do Município de São Paulo [updated 2016]. Disponível em: http://www. prefeitura.sp.gov.br/cidade/secretarias/saude/saude_da mulher/index.php? $\mathrm{p}=5778$.

3. Brasil. Ministério da Saúde. Secretaria de Assistência à Saúde. Departamento de Assistência e Promoção à Saúde. Coordenação Materno Infantil. Manual dos comitês de mortalidade materna. Brasília: Ministério da Saúde; 1994. efetiva redução da mortalidade materna em nosso meio é simples, porém de difícil execução.

O exercício da medicina em toda a sua plenitude, respeitando os Protocolos Assistenciais e com a utilização da medicina baseada em evidências é providência suficiente para atingirmos nossos objetivos.

Não entendam a "medicina" como "médico". Todos os profissionais que prestam atendimento à saúde têm responsabilidade equânime. Se cada profissional exercer sua atividade em sintonia, respeitando e se atendo aos preceitos que sua área de atuação determina, caminharemos na direção certa.

Identificamos falhas na ORIENTAÇÃO do momento apropriado para gestar, na PREVENÇÃO E TRATAMENTO das comorbidades, na COMUNICAÇÃO entre o profissional e o paciente e mesmo entre profissionais, na observância aos PROTOCOLOS ASSISTENCIAIS e, finalmente, descaso com o paciente, que merece um TRATAMENTO DIGNO, diretamente vinculado a nossa postura profissional, dentro de um ambiente que forneça condição minimamente humana para prestarmos esse atendimento.

O fornecimento da medicação e dos cuidados apropriados a cada caso, a formação e a atualização profissional, o incremento de locais para o atendimento à gestação de risco, a constituição de equipes completas para o atendimento hospitalar, a cobertura abrangente da rede assistencial, o fortalecimento de um sistema hierarquizado de atendimento à saúde, bancos de sangue eficientes, Comissões de Auditoria, educação de qualidade, saneamento básico e transportes são fatores importantes e coadjuvantes para alcançarmos nosso objetivo.

Como vimos, a morte materna é uma ocorrência multicausal. A responsabilidade para sua redução é de todos nós, profissionais da saúde, população atendida, educadores, professores, sociólogos e políticos. Reflete não só a perda de um ente querido como também, toda a desestrutura de um povo. Somente o envolvimento conjunto de todas as esferas poderá contribuir para obtermos uma saúde digna e de qualidade.

4. Vega CEP. Formulário para notificação de óbito materno. São Paulo: Secretaria da Saúde do Município de São Paulo; 2017. Disponível em: http://www9.prefeitura.sp.gov.br/forms/obito/ formObito.php.

5. Vega CEP, Santos JLdO, Marcus PAF, Castro Filho JMd, Terra CdM. O impacto da pandemia por influenza A (H1N1) na mortalidade materna do Município de São Paulo - 2009. Disponível em: http://www.prefeitura.sp.gov.br/cidade/ secretarias/saude/saude_da_mulher/index.php? $\mathrm{p}=5778$.

6. O'Gorman N, Wright D, Syngelaki A, Akolekar R, Wright A, Poon LC, et al. Competing risks model in screening for preeclampsia by maternal factors and biomarkers at 11-13 
weeks gestation. Am J Obstet Gynecol. 2016;214(1):103. e1-.e12. doi: 10.1016/j.ajog.2015.08.034.

7. Rolnik DL, Wright D, Poon LCY, Syngelaki A, O'Gorman N, de Paco Matallana C, et al. ASPRE trial: performance of screening for preterm pre-eclampsia. Ultrasound Obstet Gynecol. 2017;50(4):492-5. doi: 10.1002/uog.18816.

8. Hofmeyr GJ, Manyame S. Calcium supplementation commencing before or early in pregnancy, or food fortification with calcium, for preventing hypertensive disorders of pregnancy. Cochrane Database Syst Rev. 2017;9:CD011192. doi: 10.1002/14651858.CD011192.pub2.

9. Oliveira MHNd, Caetano Costa MEN, Toscano PRP, Tedoldi CL. Fármacos cardiovasculares na gestação e amamentação. Arq Bras Cardiol. 2009;93(6 supl.1):120-6. http://dx.doi. org/10.1590/S0066-782X2009001300006.

10. Schorn MN, Phillippi JC. Volume replacement following severe postpartum hemorrhage. J Midwifery Womens Health. 2014;59(3):336-43. doi: 10.1111/jmwh.12186.

11. Lira Plascencia J, Ibarguengoitia Ochoa F, Argueta Zuniga M, Karchmer S. Placenta praevia/accreta and previous cesarean section. Experience of five years at the Mexico National Institute of Perinatalogy. Ginecol Obstet Mex. 1995;63:33740.

12. Abu-Heija AT, El-Jallad F, Ziadeh S. Placenta previa: effect of age, gravidity, parity and previous caesarean section. Gynecol
Obstet Invest. 1999;47(1):6-8. doi: 10.1159/000010053.

13. Aleixo Neto A. Smoking effects on pregnancy. Rev Saude Publica. 1990;24(5):420-4.

14. Haghenbeck-Altamirano FJ, Leis-Márquez T, Ayala-Yáñez $\mathrm{R}$, Juárez-García LeC, García-Moreno C. Antenatal diagnosis of placental acretism-percretism. Ginecol Obstet Mex. 2013;81(5):259-71.

15. Andrade BAM, Gagliardo GI, Péret FJA. Tromboembolismo venoso no ciclo gravídico puerperal. Femina. 2009;37(11):611-18. Disponível em: http://files.bvs.br/ upload/S/0100-7254/2009/v37n11/a004.pdf.

16. Lage EM, Barbosa AS. Cardiopatias e gravidez. Femina. 2012;40(1):43-50. Disponível em: http://files.bvs.br/ upload/S/0100-7254/2012/v40n1/a3079.pdf.

17. Sebastião AM, coordenador. Recomendações da SOCERJ em cardiopatia e gestação. Rev SOCERJ. 2004;17(Supl.C):1-40. Disponível em: http://sociedades.cardiol.br/socerj/ revista/2004_supl_c/a2004_v17_disseccao_aguda\%20.pdf

18. Smok DA. Aortopathy in pregnancy. Semin Perinatol. 2014;38(5):295-303. doi: 10.1053/j.semperi.2014.04.019.

19. Laurenti R, Mello Jorge MHP, Vega CEP. Morbidade materna em adolescentes - gravidez de adolescentes. São Paulo: Faculdade de Saúde Pública da Universidade de São Paulo; 2009. Disponível em: http://www.prefeitura.sp.gov.br/cidade/ secretarias/saude/saude_da_mulher/index.php?p=5778. 